\title{
Resistive Switching of Carbon-Based RRAM with CNT Electrodes for Ultra-Dense Memory
}

\author{
Yang Chai ${ }^{\dagger \dagger}$ Yi Wu ${ }^{\dagger}$, Kuniharu Takei ${ }^{*}$, Hong-Yu Chen ${ }^{\dagger}$, Shimeng Yư ${ }^{\dagger}$, Philip C. H. Chan ${ }^{\ddagger \S}$, Ali Javey \\ and H. -S. Philip Wong ${ }^{\dagger}$
}

${ }^{\dagger}$ Department of Electrical Engineering and Center for Integrated Systems, Stanford University, USA

"Department of Electronic and Computer Engineering, Hong Kong University of Science and Technology, Hong Kong, China

"Department of Electrical Engineering and Computer Sciences, University of California at Berkeley, USA

${ }^{\S}$ Department of Electronic and Information Engineering, The Hong Kong Polytechnic University, Hong Kong, China

Tel.: (1)-650-283-0290, Email: yangchai@stanfrord.edu

\begin{abstract}
We demonstrate the nonvolatile resistive switching of an amorphous carbon $(\mathrm{a}-\mathrm{C})$ layer with carbon nanotube (CNT) electrodes for ultra-dense memory. The use of CNT as electrode leads to the ultimately scaled cross-point area $(\sim 1$ $\mathrm{nm}^{2}$ ), and the use of a-C results in an all-carbon memory. Carbon-based complementary resistive switching (CRS) is shown for the first time, enabling cross-point memory without cell selection devices.
\end{abstract}

\section{Introduction}

Amorphous carbon $(\mathrm{a}-\mathrm{C})$ is a non-crystalline carbon allotrope in which the long range crystalline order is not present. The a-C has been shown to exhibit resistive switching behavior for nonvolatile memory application [1-5]. Different switching mechanisms for the a-C have been reported, including thermo-chemical $\mathrm{sp}^{2}$ carbon chain forming/rupture [1-3] electrochemical metallization [4] and valence change [5].

This work demonstrates nonvolatile resistive switching for an a-C layer, sandwiched between top metal electrode and bottom CNTs. The single-walled CNT, with an average diameter of $1.2 \mathrm{~nm}$, is the ideal nanoscale electrode for ultra-dense memory cells. Complementary resistive switching (CRS) [6] for a planar metal/a-C/CNT/a-C/metal device is demonstrated for the first time. This CRS device enables large passive crossbar array with the CRS itself serving as an integrated array cell selection device. As a look-ahead experiment for ultimately scaled cell area $\left(1.2 \times 1.2 \mathrm{~nm}^{2}\right)$, we show preliminary data for an all-carbon $\mathrm{CNT} / \mathrm{a}-\mathrm{C} / \mathrm{CNT}$ device, in which the switching element is the a-C layer sandwiched between a set of double transferred CNT crossbar electrodes. These results show the potential of all-carbon device and CNT electrodes for ultra-dense memory.

\section{Device Fabrication and Material Characterization}

Fig. 1(a) and (b) show the process flow for fabricating the metal/a-C/CNT/metal device and metal/a-C/CNT/a-C/metal device. Horizontally aligned CNT were grown on quartz substrate and were transferred to $\mathrm{Si} / \mathrm{SiO}_{2}$ substrate using the process in [7]. The average CNT density is $\sim 2 / \mu \mathrm{m}$. The a-C pattern was defined by lithography. A 30-nm thick a-C layer was deposited by e-beam evaporation, followed by lift-off. The top metal electrodes were patterned by a second e-beam evaporation and lift-off. The unwanted CNT were etched by oxygen plasma, leaving the CNT only in the active region. Fig. 2 shows an SEM image of the fabricated devices. The sheet resistivity $(121.7 \pm 75.7 \Omega \cdot \mu \mathrm{m})$ of the a-C was determined by four-point electrical measurement. The a-C produced by condensation onto a substrate from energetic carbon ion fluxes is rich with $\mathrm{sp}^{3}$ bonding [8]. Fig. 3 shows the XPS spectrum of the as-deposited a-C film. No metal atoms were doped into the a-C film during the deposition.


Fig. 1. Process flow for fabricating carbon-based memory cell. (a) Metal/a-C/CNT/metal with asymmetric CNT contact structure. A 30nm-thick a-C was deposited on top of one end of the CNT. A $60 \mathrm{~nm}$-thick metal was then deposited on both ends of the CNT. (b) Metal/a-C/CNT/a-C/Metal with symmetric CNT contact structure. Both ends of the CNT are contacted with $\mathrm{metal} / \mathrm{a}-\mathrm{C}$.

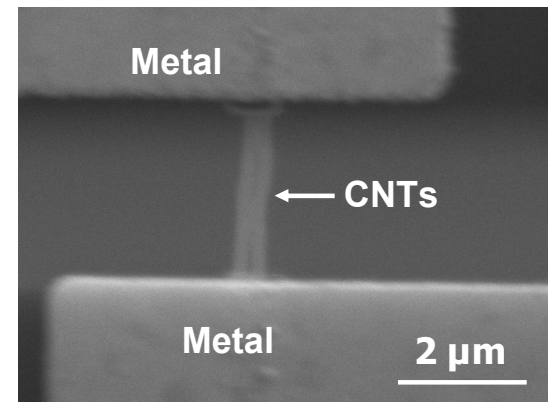

Fig. 2. An SEM image of a fabricated device. The CNT acts as bottom electrode for the memory cell. The width of the typical device is $1 \mu \mathrm{m}$. The number of the CNTs varies from 1-3. 


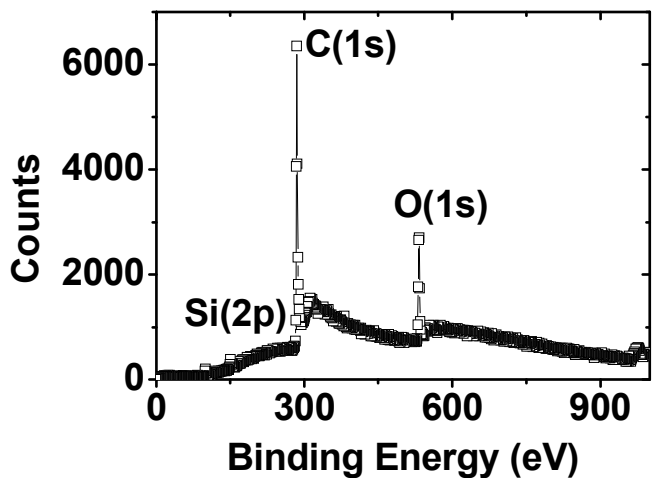

Fig. 3. XPS spectrum of the as-deposited $30-\mathrm{nm}$ thick a-C. No metal signal is detected from the spectrum, indicating that metal atoms were not doped in the a-C film.

Switching Behavior of Metal/a-C/CNT/Metal Device

\section{A. Bipolar Switching with Fast Diffusing Metal}

The top metal plays an important role on determining the switching behavior of the a-C. The fast diffusing metal such as $\mathrm{Au}, \mathrm{Ag}$, and $\mathrm{Cu}$ on top of a-C have been reported to exhibit electrochemical metallization bipolar switching behavior [4], in which the cell was switched ON by applying a positive bias and switched OFF only by applying the opposite bias. Fig. 4 shows the typical I-V curve of an $\mathrm{Au} / \mathrm{a}-\mathrm{C} / \mathrm{CNT} / \mathrm{Au}$ cell, exhibiting bipolar switching behavior. The current-voltage relationship indicates the different conduction mechanism in high resistance state (HRS) and low resistance state (LRS). The I-V curve of the LRS state of $\mathrm{Au} / \mathrm{a}-\mathrm{C} / \mathrm{CNT} / \mathrm{Au}$ cell shows an Ohmic-like behavior with a slope of 1.1. This is believed to be resulted from the formation of $\mathrm{Au}$ and $\mathrm{Ag}$ conduction filament during the SET process. The charge transport in HRS is in agreement with a trap-controlled space charge limited current mechanism. The $\mathrm{Ag} / \mathrm{a}-\mathrm{C} / \mathrm{CNT} / \mathrm{Ag}$ device shows similar behaviors to the $\mathrm{Au} / \mathrm{a}-\mathrm{C} / \mathrm{CNT} / \mathrm{Au}$ device (Fig. 4(b)). Fig. 5(a) shows the DC sweep endurance measurement of an $\mathrm{Au} / \mathrm{a}-\mathrm{C} / \mathrm{CNT} / \mathrm{Au}$ device. The memory cell successfully operates over 27 times. The $\mathrm{OFF} / \mathrm{ON}$ ratio is over 80 . The evolution of the voltage of the SET and RESET is shown in Fig. 5(b). The $\mathrm{V}_{\text {SET }}$ and $\mathrm{V}_{\text {RESET }}$ of metal/a-C/CNT/metal are higher than those of metal/a-C/metal structure [4], probably related to the high series resistance of bottom $\mathrm{CNT} /$ metal contact. The ON and OFF state of the memory cells retained for at least two weeks at room temperature in air, indicating the nonvolatile property of the memory cell.

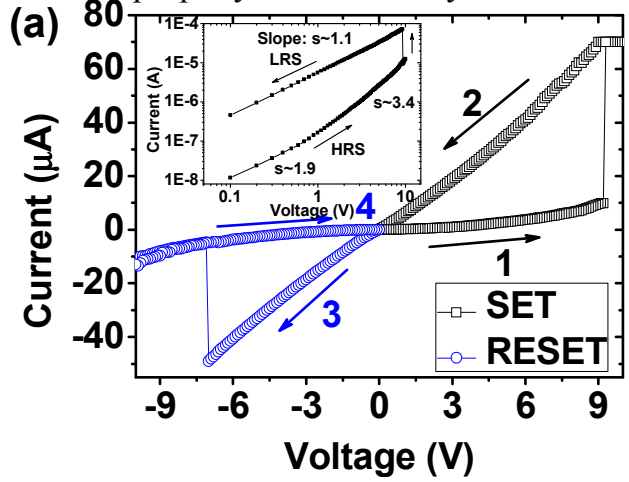

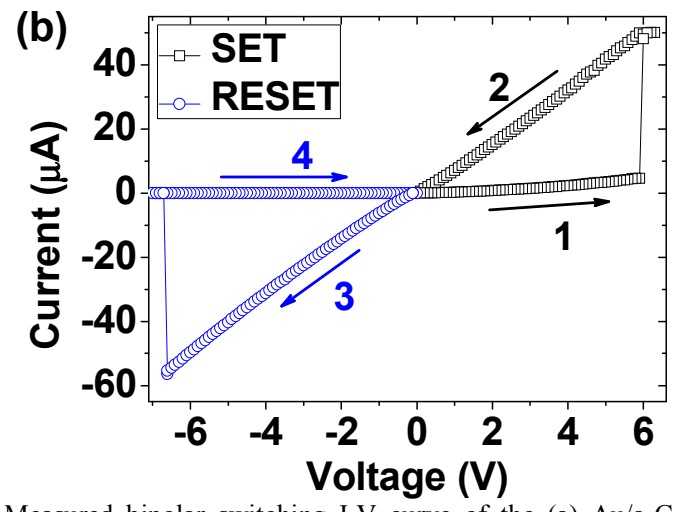

Fig. 4. Measured bipolar switching $\mathrm{I}-\mathrm{V}$ curve of the (a) $\mathrm{Au} / \mathrm{a}-\mathrm{C} / \mathrm{CNT} / \mathrm{Au}$ $(60 \mathrm{~nm} / 30 \mathrm{~nm} / \mathrm{CNT} / 60 \mathrm{~nm})$ device. Inset: $\log -\log$ plot. The HRS and LRS state exhibit different conduction mechanisms. The LRS I-V has a nearly linear $\mathrm{I}-\mathrm{V}$ relationship. The charge transport in HRS is in agreement with a trap-controlled space charge limited current mechanism. Reset current is about $70 \mu \mathrm{A}$. (b) $\mathrm{Ag} / \mathrm{a}-\mathrm{C} / \mathrm{CNT} / \mathrm{Ag}$ device.



(b)

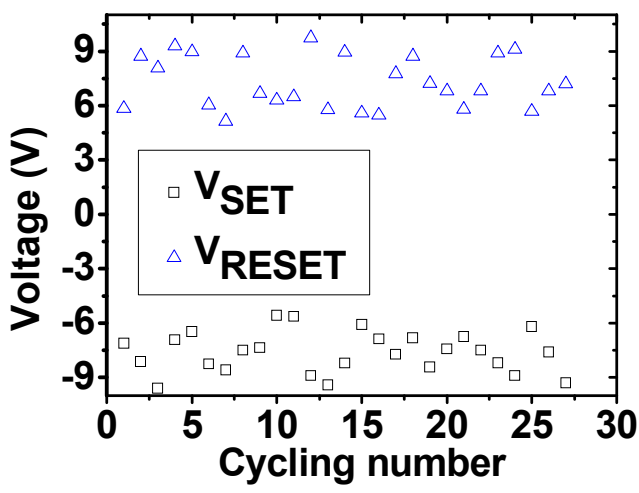

Fig. 5. (a) Endurance measurement of an $\mathrm{Au} / \mathrm{a}-\mathrm{C} / \mathrm{CNT} / \mathrm{Au}$ cell. The resistance value was read at $100 \mathrm{mV}$. The memory cell successfully operates over 27 times. (b) Evolution of $\mathrm{V}_{\text {set }}$ and $\mathrm{V}_{\text {reset }}$ as a function of the switching cycle.

\section{B. Unipolar Switching with Inert Metal}

Unipolar switching has been reported for a-C layer with an inert metal electrode, such as, $\mathrm{W}$ and $\mathrm{Cr}[1,3]$. In our work, we used $\mathrm{Pd}$ as top metal electrode, which typically forms good electrical contact to the CNT. Fig. 6(a) shows one SET and RESET I-V curves from the Pd/a-C/CNT/Pd memory cell, exhibiting the unipolar switching behavior. The a-C consists of both $\mathrm{sp}^{2}$ hybridized bonds and $\mathrm{sp}^{3}$ hybridized bonds. The 
$\mathrm{sp}^{2}$ bonding-dominated graphitic form has low resistance close to that of a metal, and the $\mathrm{sp}^{3}$ bonding-dominated diamond form has high resistance. The transformation between $\mathrm{sp}^{2}$ and $\mathrm{sp}^{3}$ carbon bonding typically involves high energy reaction and catalyzed metal $[2,9]$. The DC sweep and pulse programming are less likely to change the chemical bonding. We speculate that the unipolar switching is the result of the atomic migration induced by external electrical force, forming a conductive $\mathrm{sp}^{2}$ carbon filament in the insulating $\mathrm{sp}^{3}$ carbon matrix (Fig. 7). In contrast to electrochemical metallization bipolar switching in a-C and other switching material, carbon is the mono-atom for both the conductive filament and the insulating matrix.

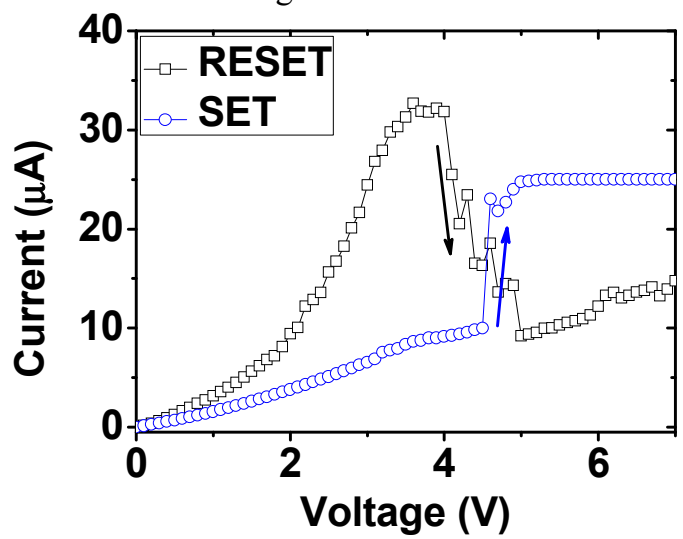

Fig. 6. One unipolar switching $\mathrm{I}-\mathrm{V}$ curves from the $\mathrm{Pd} / \mathrm{a}-\mathrm{C} / \mathrm{CNT} / \mathrm{Pd}$ device. The device is SET around $4.7 \mathrm{~V}$ and RESET around $3.8 \mathrm{~V}$.

\section{$\mathrm{sp}^{2}$ bond carbon}

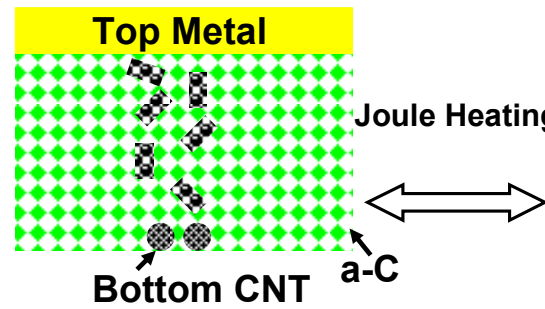

Fig. 7. Schematic of the unipolar switching mechanism of a-C. The unipolar switching for a-C with inert metal electrode is resulted from the atomic migration induced by external electrical force (possibly the Joule heating), forming a conductive $\mathrm{sp}^{2}$ carbon filament in the insulating $\mathrm{sp}^{3}$ carbon matrix.

\section{Switching Behavior of Metal/a-C/CNT/a-C/Metal Device}

Cross-point memory, where the bi-stable material is sandwiched between parallel bottom electrode and perpendicular top electrode, has been proposed for ultra high density memory, because the memory cell size can be reduced to $4 \mathrm{~F}^{2}(\mathrm{~F}=$ minimize feature size $)$, and potentially even smaller if the memory cells are stacked in 3D. However, the sneak current in the cross-point memory is an inherent disadvantage [6]. A selection diode integrated with the memory cell is often proposed to solve the sneak current path problem [10].
Recently, complementary resistive switches (CRS) have been proposed for solving the sneak leakage current in cross-point memory [6]. We fabricated carbon-based lateral CRS structure with two bipolar metal/a-C/CNT/metal cells connected by a common CNT in anti-series as a metal/a-C/CNT/a-CNT/metal device (Fig. 1(b)). Fig. 8 shows typical I-V curves of a Au/a-C/CNT/a-C/Au cell, which is a superimposed I-V characteristic of the two bipolar memory cells in anti-series. The 4 distinct threshold voltages enable us to define the CRS cell with 4 different states ["ON" (LRS/LRS), "OFF" (HRS/HRS), "0" (LRS/HRS) and "1" (HRS/LRS)] [6]. The bit information is stored in the two anti-serial memory cells, while the overall resistance of the CRS remains the dominated by the HRS $\left(\mathrm{R}_{\mathrm{HRS}}\right)$. The CRS cell exhibits HRS when storing the bit information, thus a passive cross-point array of CRS cells does not require memory cell selection devices. This resistive switching cell without the selective device enables high memory density. The two distinct HRS states storing the bit information also reduce the static power consumption [6].

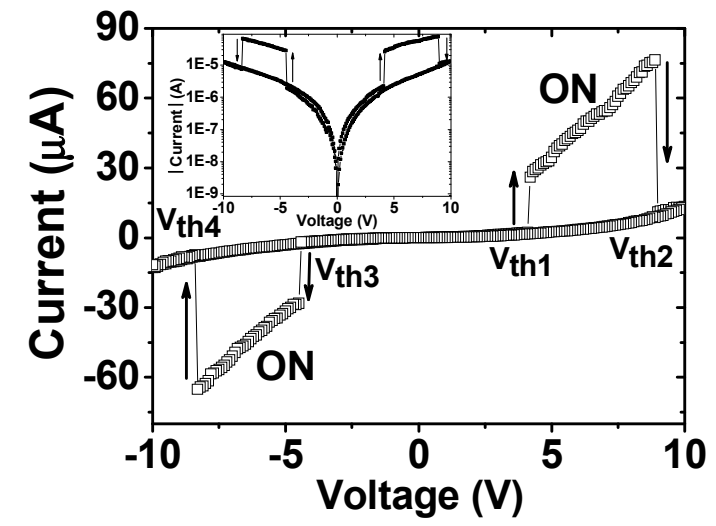

Fig. 8. Measured $\mathrm{I}-\mathrm{V}$ curves of the two bipolar memory $(\mathrm{Au} / \mathrm{a}-\mathrm{C} / \mathrm{CNT} / \mathrm{a}-\mathrm{C} / \mathrm{Au})$ cells in anti-series (CRS). The combined cell shows "ON" state when both cells are in LRS state, and the "OFF" state when both cells are in HRS state. The bit information " 0 " and " 1 " state can be defined as one cell is in HRS, and the other in LRS state, respectively. A passive cross-point array of CRS cells does not require memory cell selection devices.

\section{CNT/a-C/CNT Device}

As a proof of concept for an ultimately scaled RRAM with truly nanoscale electrodes, we fabricated a CNT/a-C/CNT device. Device fabrication is similar to the metal/a-C/CNT/metal device except that after depositing the a-C memory layer, another set of aligned CNT was transferred onto the cell with the CNT orthogonal to the CNT underneath (Fig. 9). The a-C layer was thus sandwiched between double transferred CNTs (Fig. 10). Each cross-point of this structure is only $1.2 \times 1.2 \mathrm{~nm}^{2}$. However, the surface of the as-deposited a-C layer is rough, resulting in the situation where part of the top CNT transfer is not successful. This leads to the low yield of the fabricated devices. 

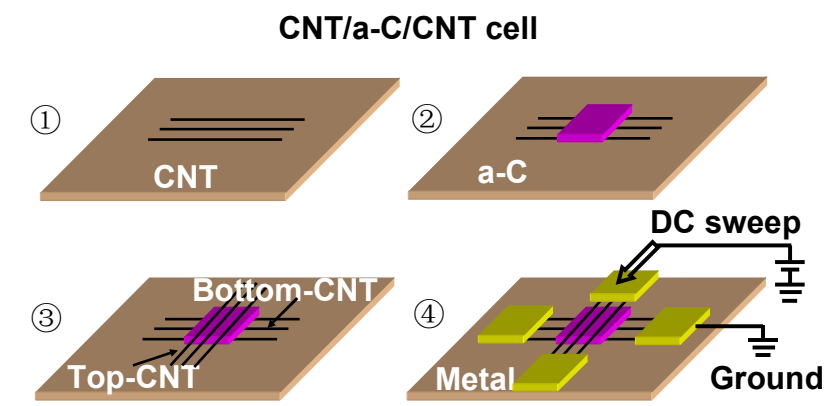

Fig. 9. Process flow for fabricating the CNT/a-CNT/CNT cell. The a-C layer was sandwiched between two orthogonally transferred CNTs. The unwanted a-C and CNT were removed by oxygen plasma etching.

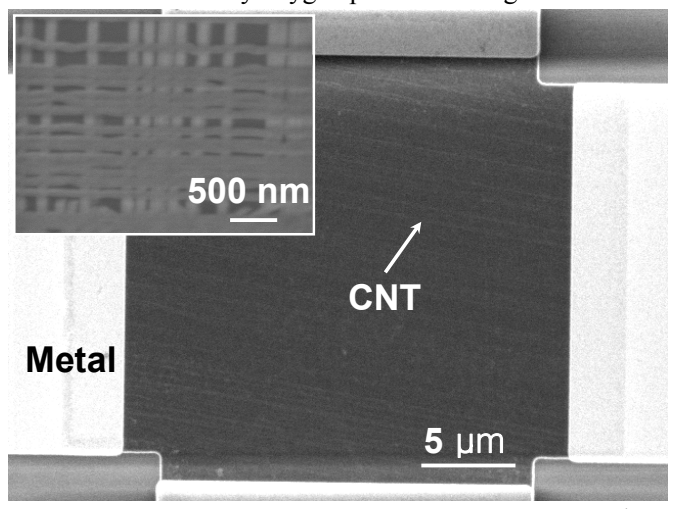

Fig. 10. SEM image of the CNT/a-C/CNT device. Only the $2^{\text {nd }}$ horizontally transferred CNTs on top of the a-C layer can be observed in the image. To show the successful double CNT transfer process, a SEM image of the CNT crossbar without a-C layer is also shown in the inset.

Fig. 11 shows the typical I-V curve from a $20 \mu \mathrm{m}$ by $20 \mu \mathrm{m}$ CNT/a-C/CNT structure ( 400 metallic CNT cross-points). The I-V curves exhibit the diode-like rectifying characteristics. We speculate that the rectifying characteristic results from imperfect contact between the a-C and the top transferred CNT. The roughness of the a-C possibly causes unknown physical and electrical barrier between top CNT and

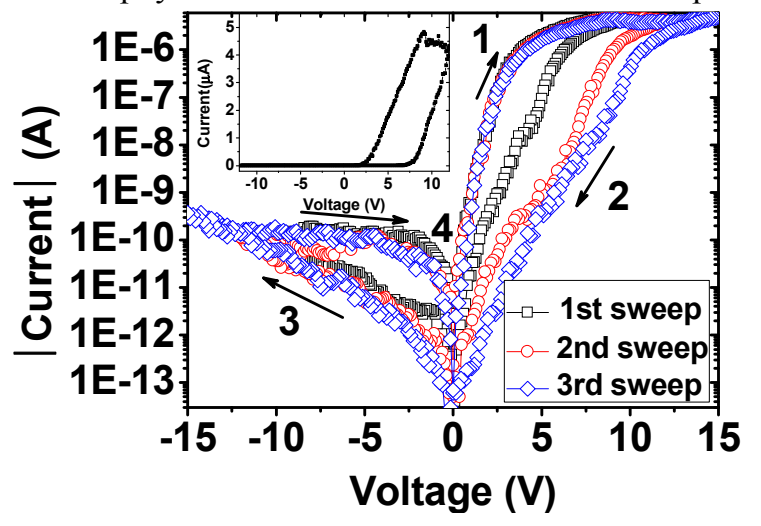

Fig. 11. Measured consecutive $I-V$ curves of a $20 \mu \mathrm{m}$ by $20 \mu \mathrm{m}$ CNT/a-C/CNT cell with about 400 metallic CNT/a-C/CNT cross-points. Imperfect contact between the top transferred $\mathrm{CNT}$ and a-C results in a rectifying characteristic. Inset: I-V curve in linear scale.
a-C. A memory window in the positive voltage branch is observed with programming current of $\sim 6 \mathrm{uA}$.

\section{Conclusion}

New carbon-based RRAM is successfully demonstrated. The use of CNT as electrode leads to the ultimately scaled cross-point area $\left(\sim 1 \mathrm{~nm}^{2}\right)$ and the use of a-C enable an all-carbon memory technology. Carbon-based CRS is shown for the first time, enabling dense cross-point memory without cell selection devices.

\section{Acknowledgement}

This work is supported in part by member companies of Stanford NMTRI, SGF, Fellowship from the O.G. Villard Engineering Fund at Stanford, the Research Grant Council of Hong Kong Government under CERG grant HKUST 611307, and the Berkeley Sensors and Actuators Center (BSAC).

\section{References}

[1] F. Kreupl, R. Bruchhaus, P. Majewski, J. B. Philipp, R. Symanczyk, T. Happ, C. Arndt, M. Vogt, R. Zimmermann, A. Buerke, A. P. Graham, and M. Kund, "Carbon-based resistive memory," IEEE IEDM Technical Digest, pp. 521-524, December 2008.

[2] X. Guan, Y. He, L. Zhao, J. Zhang, Y. Wang, H. Qian, and Z. P. Yu, "Simulation study of switching mechanisms in carbon-based resistive memory with molecular dynamics and extended Hukel theory-based NEGF method," IEEE IEDM Technical Digest, pp. 905-908, December 2009.

[3] A. Sinitskii and J. M. Tour, "Lithographic graphitic memories," ACS Nano, Vol. 3, No. 9, pp. 2760-2766, August 2009.

[4] F. Zhuge, W. Dai, C. L. He., A. Y. Wang, Y. W. Liu, M. Li, Y. H. Wu, P. Cui, and R. W. Li, "Nonvolatile resistive switching memory based on amorphous carbon," Appl. Phys. Lett., Vol. 96, pp. 163505-163507, April 2010.

[5] E. G. Gerstner and D. R. Mckenzie, "Nonvolatile memory effects in nitrogen doped tetrahedral amorphous caron thin films," J. Appl. Phys., Vol. 84, No. 10, pp. 5647-5651, August 1998.

[6] E. Linn, R. Rosezin, C. Kugeler, and R. Waser, "Complementary resistive switches for passive nanocrossbar memories," Nature Materials, Vol. 9, pp. 403-406, May 2010.

[7] N. Patil, A. Lin, E. R. Myers, K. Ryu, A. Badmave, C. W. Zhou, H-. S. P. Wong and S. Mitra, "Wafer-scale growth and transfer of aligned sigle-walled carbon nanotubes," IEEE Trans. Nanotechnology, Vol. 8, No. 4, pp. 498-504, July 2009 .

[8] S. Bhattacharyya, S. J. Henley, E. Mendoza, L. Gomez-Rojas, J. Allam, S. R. P. Silva, "Resonant tunnelling and fast switching in amorphous-carbon quantum-well structures," Nature Materials, Vol. 5, No. 1, pp. 19-22, January 2006.

[9] M. Zheng, K. Take, B. Hsia, H. Fang, X. B. Zhang, N. Ferralis, H. Ko, Y. L. Chueh, Y. G. Zhang, R. Maboudian and A. Javey, "Metal-catalyzed crystallization of amorphous carbon to graphene," Appl. Phys. Lett., Vol. 96, pp. 063110-063112, February 2010.

[10] J. Liang and H.-S. P. Wong, "Size Limitation of Cross-Point Memory Array and Its Dependence on Data Storage Pattern and Device Parameters," IEEE International Interconnect Technology Conference Burlingame, California, USA, pp. 6.3, June, 2010. 